\title{
LA CONFIGURACIÓN DE ESPACIOS \\ FRACTALES EN LA MÚSICA MINIMALISTA
}

\author{
Diego Franco ${ }^{(a)}$
}

CONFIGURATION OF FRACTAL SPACES IN MINIMAL MUSIC

A CONFIGURAÇÃO DOS ESPAÇOS FRACTAIS NA MÚSICA MINIMALISTA

Fecha de recepción: 28 de enero del 2019

Fecha de aprobación: 9 de febrero del 2019

Disponible en línea: 22 de febrero del 2019

\section{Sugerencia de citación:}

Franco, D. (2019). La configuración de espacios fractales en la música minimalista. Razón Crítica, 6, 133154, doi: $10.21789 / 25007807.1451$

\author{
(a) Diego Franco \\ Profesional en Estudios Musicales de la Pontificia Universidad Javeriana y \\ Especialista en Gerencia de Empresas, Servicios y \\ Productos de la música de la Universidad EAN \\ Profesor del Departamento de Comunicación Social y Cinematografía de la \\ Universidad de Bogotá Jorge Tadeo Lozano, Colombia \\ https://orcid.org/0000-0003-4544-0491 \\ diegoh.francoc@utadeo.edu.co
}




\section{R E S U M E N}

Teniendo en cuenta la importancia de la repetición en el minimalismo y la recurrencia del uso reiterativo de motivos musicales en las formas clásicas, se pretende en este ensayo, en primera instancia, establecer una genealogía de la repetición a lo largo de la historia de la música, para luego ver de qué forma las formas recursivas encuentran su mayor expresión en el minimalismo. Se realizará un análisis sobre la notación, la caligrafía y la gramática musical, y se indagará sobre la forma en que estas fueron el objeto de profundas transformaciones durante la Segunda Revolución Industrial. Se verá también cómo estas transformaciones permitieron una reformulación tanto en la notación musical, como de la misma definición de música. En la última parte de este ensayo, a partir de la teoría de la narración fractal de German A. Duarte, se propondrá un análisis de las obras más características del minimalismo en la música para de qué manera la música para minimalista configura espacios fractales.

PALABRAS CLAVE: minimalismo, fractal, repetición, música, representación 


\section{A B S T R A C T}

Taking into account the importance of repetition in minimalism and the frequency in the reiterative use of musical motifs in classical forms, this essay tries first to establish a genealogy of repetition throughout the history of music to later explore how recursive forms find their greatest expression in minimalism. We will carry out an analysis on notation, calligraphy and musical grammar, and study the way in which these elements deeply changed during the technological acceleration of the second industrial revolution. We will also see how these transformations allowed a reformulation of musical notation and the definition of music itself. In the last part of this essay, based on the theory of fractal narration by German A. Duarte, we will propose an analysis of the most characteristic works of minimalism in music and address how minimal music shapes fractal spaces.

KEY WORDS: Minimalism, fractal, repetition, music, representation

\section{R E S U M O}

Diante da importância da repetição no minimalismo e da recorrência ao uso repetitivo de motivos musicais em formas clássicas, este ensaio pretende, em primeiro lugar, estabelecer uma genealogia da repetição ao longo da história da música. Veremos como as formas recursivas encontram sua maior expressão no minimalismo. Realizaremos uma análise sobre a notação, a caligrafia e a gramática musical para indagar como elas se tornaram objeto de profundas transformações durante a aceleração tecnológica da segunda revolução industrial. Também veremos como essas transformações permitiram uma reformulação tanto na notação musical quanto na definição da própria música. $\mathrm{Na}$ última parte deste ensaio, a partir da teoria da narração fractal de German A. Duarte, analisaremos as obras mais características do minimalismo na música e veremos como a música minimalista configura espaços fractais.

PALAVRAS-CHAVE: Minimalismo, fractal, repetição, música, representação 


\section{La música como medio de representación y la transformación en la notación a partir de la innovación tecnológica}

Según algunos antropólogos y etnomusicólogos, el homo sapiens, identificando las formas de comunicación de otras especies — por ejemplo, de los primates, los felinos y las aves que eran capaces de transmitir mensajes cubriendo grandes distancias mediante la emisión de sonidos sin utilizar nada más que sus órganos vocales-, inició un proceso de mímesis que lo habría llevado al desarrollo de instrumentos que, como toda tecnología, suplían la debilidad natural de los sentidos. Dichos instrumentos facilitaron la comunicación entre diferentes comunidades, colmando así las distancias que las dividían. Surgieron de esta forma algunos sistemas de comunicación sonora que no solo complementaban a los sistemas visuales ${ }^{1}$ sino que también le dieron origen a las primeras formas de comunicación musical. Una vez que estos conjuntos de códigos sonoros entraron en el proceso comunicativo, y en consecuencia cultural, empezaron a ser memorizados y conservados por diferentes sociedades. Varias son las muestras arqueológicas, tanto en Occidente como Oriente, que evidencian la existencia de una notación musical rupestre. Sin embargo, el Epitafio de Seikilos es considerado todavía en Occidente como

1 La evidencia arqueológica muestra que, a pesar de no contar con sistemas efectivos de comunicación sonora, el ejercicio de encender fuego permitía configurar sistemas o códigos de comunicación básicos. 
la primera obra musical registrada de forma escrita. El Epitafio de Seikilos es un poema de amor tallado sobre un cilindro de piedra ${ }^{2}$; en este se evidencian símbolos que indican el modo y la entonación en los que cada fonema debe ser "cantado"3.

La musicología, de clara impronta eurocentrista, se ha encargado de analizar las estructuras musicales que se consolidaron a lo largo de la historia. Este campo de estudio también se ha encargado de analizar los sistemas de notación tradicionales que representan esquemas y estructuras que se han consolidado - especialmente entre la Edad Media y el siglo $\mathrm{xx}$ - en sistemas escritos de los cuales derivan diferentes formas musicales que hoy llamamos clásicas ${ }^{4}$. Se hace necesario también considerar a la tecnología, pues esta constituye un elemento esencial en la configuración de estas formas, si tenemos en cuenta la necesidad de ciertos instrumentos que requieren, una notación específica.

Las innovaciones mecánicas de la Revolución Industrial trajeron consigo aplicaciones a la música que, además de verse reflejadas en la arquitectura para la música ${ }^{5}$, afectaron considerablemente la mecánica

2 El epitafio data del primer siglo a. C. La piedra se encuentra en el Museo Nacional de Copenhague —número de inventario 14.897-.

3 Es importante considerar la evolución de la notación musical, sin importar el origen que se considere, a partir de las hipótesis de la notación rupestre postuladas por los historiadores del arte -o a partir del Epitafio de Seikilos, en Occidente- según las cuales la notación musical, de manera paralela a la escritura, ha evolucionado con base en las necesidades de ejecución sonora del momento — bien sea por las características que la música debía tener, o por la aparición de nuevos instrumentos-. Partiendo desde la notación neumática, el ars nova, las tablaturas del Renacimiento, pasando por el himno Ut queant laxis (véase Palisca y Gruot, 1996), llegamos al sistema de notación actual, con el cual, y a lo largo de los períodos del Barroco, Clasicismo, Romanticismo y el siglo xx, se escribe toda la música de occidente.

4 Las formas musicales surgen, dentro de la historia de Occidente, con base en los diferentes tratados que, en el caso de la Edad Media, estaban mediados por la religión. A partir de dichos tratados, las melodías, progresiones armónicas, tonalidades, instrumentación, etcétera, debían ser manipuladas de formas precisas. Este tipo de tratamientos dieron origen, por ejemplo, a los cantos gregorianos, y permitieron establecer las diferencias entre el uso sagrado y secular de la música. Durante el período Barroco, en el marco secular, las danzas como el minué, la gigue, el burrée, contaban con sus reglas de composición. Johan Sebastian Bach, con el Clave bien temperado, permite que la línea que separaba lo secular de lo sacro se adelgace, generando una mezcla de formas musicales que en el período Clásico desembocarían en la sonata y la sinfonía. Por su parte, el período Romántico aportaría las bases para que el siglo xx, en términos musicales, fuera recibido. Igualmente, para que durante este tiempo la transformación social y política del mundo lleguen a la música, y que las reglas consolidadas durante cuatro períodos, después de ser estudiadas y apropiadas, pudieran ser transgredidas. No hay que dejar de resaltar que lo anterior no fue ajeno a las demás formas de expresión musical como la ópera y que, incluso, brindó versatilidad al uso de la música y generó así nuevos espacios.

5 Con el perfeccionamiento de las artes performativas se incrementaron las demandas sonoras que, ante la inexistencia de sistemas de amplificación de sonido, debían ser tratadas arquitectónicamente. La aparición de instrumentos que podían generar una intensidad sonora suficientemente baja, como es el caso del piano, requirió que los públicos se acercaran al escenario y, de la misma manera, que las conchas acústicas de los escenarios fueran más eficientes en proyección sonora. Los arquitectos debían pensar en el diseño utilizando materiales que reflejarán de manera óptima el sonido a lo largo del auditorio. 
de los instrumentos. Consideremos, por ejemplo, el trombón de pistones ${ }^{6}$ o el perfeccionamiento de la mecánica del piano, que extendió su rango dinámico ${ }^{7}$ y brindó a compositores e intérpretes mayor flexibilidad creativa. Sin embargo, en este contexto tecnológico particular no solo los instrumentos fueron modificados. También vemos el desarrollo de una serie de instrumentos musicales mecánicos y juguetes sonoros tales como la caja de música, órganos portátiles y pianolas. Estos instrumentos encuentran como base tecnológica el desarrollo del cilindro codificado, un cilindro construido en madera o aluminio sobre el cual se ponía una serie de clavos, u orificios. El cilindro, al momento de girar dentro de un mecanismo temperado o afinado, generaba melodías. En el caso del órgano portátil, el cilindro era perforado con el fin de que, al girar dentro de un compresor manual de aire, el aire saliera hacia las diferentes flautas del órgano y produjera así las melodías. El principio del cilindro codificado fue aplicado a otros soportes, como el papel, las tablas perforadas e incluso la tela. La automatización o mecanización en la ejecución de diversos instrumentos generó de cierta forma un tipo de enajenación del intérprete-ejecutor. En un fenómeno análogo al producido por el ingreso del daguerrotipo en la representación pictórica, esta automatización generó en la música la sensación de que la necesidad de dominar un lenguaje musical ya no era indispensable para producir música.

Las limitaciones físicas del cilindro imposibilitaban la codificación de piezas musicales de gran extensión. Una solución a dicha limitación fue la codificación de fragmentos, que básicamente eran frases cortas o pasajes de diferente duración que sucesivamente eran reproducidos

\footnotetext{
6 El trombón es un instrumento aerófono perteneciente a la familia de los metales o cobres, por su material y técnica para generar el sonido. De origen barroco, el trombón moderno produce las notas musicales variando la longitud de un tubo cerrado en el que vibra el aire con la ayuda de una vara corrediza. Para el siglo xix, a la vara se agregaron unos pistones, como en la trompeta, que le permitían al trombón generar notas adicionales sobre cada posición de la vara aumentando su tesitura.

7 El piano es una invención musical del siglo xvir, es una evolución al clavecín. El piano es un instrumento de cuerda percutida que produce el sonido generando el movimiento de una palanca que a su vez acciona un martillo cubierto con piel que golpea la cuerda. Contrario a su antecesor, el piano-forte se denomina así por la posibilidad de generar sonidos de baja o alta intensidad. En el siglo xix, el reemplazo de la piel por paños de fieltro le permitió al intérprete poder ejecutar sonidos aún más suaves. En términos de notación, la intensidad en una partitura se indica con la escritura de una letra $f$ (forte) o $p$ (piano) sobre determinada nota en el pentagrama. El incremento en la cantidad de letras determinaba el nivel de intensidad; por ejemplo, fff (fortissimo) indicaba una ejecución con la mayor intensidad sonora posible. La adición de fieltro a los martillos permitió que los compositores pudieran adicionar una cuarta letra a estas indicaciones, a tal punto de superar el pianissimo $(p p p)$ con un molto pianissimo $(p p p p)$. La aparición de este tipo de elementos dio paso a que en la música se pudieran escribir piezas con una mayor carga emocional, siendo está una de las causas que dieron paso a la aparición de la música romántica del siglo xıx.
} 
repetidamente. Esta repetición se hacía evidente en instrumentos como el órgano portátil, un instrumento de manivela que, terciado por un sujeto —el "organista" - , repetía el tema musical una y otra vez.

Podría parecer que las formas recursivas ingresaron en la esfera de la expresión musical con los mecanismos de automatización. Cabe recordar, sin embargo, que la recursión siempre ha sido un instrumento de expresión en música. Las formas clásicas como la sonata, el rondó, las suites de danzas barrocas y la canción ejercen la recursión en el retorno. Es decir, para los compositores clásicos también era necesario poder retomar los temas, repetirlos con variaciones sustanciales que generaran contraste entre las diferentes partes de la pieza. La quinta sinfonía de Beethoven, por ejemplo, basa sus cuatro movimientos en el tema configurado por las tres corcheas seguidas de una negra [ta-ta-ta-tán]. Si se escuchan con atención los tres movimientos que siguen al ya popular primer movimiento, el análisis permite ver una recurrencia en el uso de estas cuatro figuras musicales, con una variación en su configuración melódica, la velocidad de ejecución (carácter), la tonalidad o el timbre. Estas variaciones que son necesarias en el estilo clásico, sin embargo, comenzaron a ser transgredidas, junto con otras "normas de escritura", como resultado de la nueva forma mentis industrial del siglo xx. Como se verá más adelante, estas transgresiones harán parte de la innovación en el uso de recursos musicales.

\section{Minimalismo: antecedentes, los nuevos recursos musicales}

El final del siglo xıx, marcado por el pleno auge de la Segunda Revolución Industrial y, en consecuencia, el ingreso de nuevas tecnologías a varios campos de la sociedad, representó un período de ruptura con respecto a las formas de producción establecidas. En el campo de la música, esa ruptura también se dio; y se manifestó especialmente por la tangible aceleración y multiplicación de la experimentación y explotación de los recursos musicales por parte de los compositores. Esta fase de experimentación encuentra sus raíces en compositores como Wolf, Debussy, Strauss, Musorgsky, Rimsky-Korsakon, quienes venían explorando otras alternativas de uso de los elementos sonoros para sus composiciones. De este rico periodo se destaca, sin lugar a duda, la obra de Gustav Mahler. 
Las sinfonías de Mahler, así como las de Bruckner, son largas, formalmente complejas y programáticas. Ellas requieren enormes grupos de intérpretes. La segunda sinfonía, estrenada en 1895, requiere una gran sección de cuerdas, diecisiete maderas, veinticinco cobres, seis timbales y otros instrumentos de percusión, cuatro o más arpas y un órgano; en adición, solistas soprano y alto y un coro grande. La octava, compuesta entre 1906-1907, y popularmente conocida como la sinfonía de mil, demanda un arreglo aún más grande de instrumentistas y cantantes. Pero el tamaño de la orquesta solo cuenta una parte de la historia. (Palisca y Grout, 1996, p. 654) ${ }^{8}$.

Como anota Donald Jay Grout, "Mahler mostró gran imaginación y osadía combinando instrumentos” (Palisca y Grout, 1996, p. 654). Es interesante resaltar el uso de la palabra 'osadía', pues esta hace referencia a la necesidad, no solo de Mahler, sino de los demás compositores del momento, de buscar un uso alternativo a la forma; de la misma manera que otros artistas, en diferentes disciplinas, lo estaban haciendo. Si contextualizamos esa 'osadía', encontramos el motor de esta en la Gran Guerra de 1914 que, antes de ver su final en 1918, daría inicio a la Revolución Rusa - pero, sobre todo, a la guerra moderna, a la guerra tecnológica, a la guerra química, esa de la masificación de la muerte- Ese período de muerte en masa que también mostró, en palabras de Grout en su Historia de la música de Occidente,

[...] experimentos radicales en el reino de la música que generaron similar inquietud y tensión en las audiencias de conciertos. Los compositores retaron a las convenciones de la tonalidad que dominaron en los siglos XVIII y xix, dando efectivamente un cierre al período Clásico-Romántico. (Palisca y Grout, 1996, p. 652).

Ciertamente, el cierre de ese gran período que marcó a la expresión artística y filosófica en su totalidad, pero sobre todo la presencia en el imaginario colectivo de la muerte mecánica, la muerte masiva de la Gran Guerra, llevaron al arte a plasmar el sentido general de una generación mutilada y traumatizada: el deseo de "no tener nada que ver" con las formas de representación existentes antes de la guerra; 
el deseo de ruptura total con el pasado, un pasado que había llevado a la guerra moderna.

La tensión política que llevó a la separación de los imperios y al fortalecimiento de las fronteras se vio reflejada en la música. La innovación musical durante el período comprendido entre 1914 y 1930 está marcada primordialmente por la transgresión de las normas clásicas; transgresión que se había consolidado en la obra de Musorgsky y Debussy, quienes habían cultivado una armonía de bloques estáticos que eliminaban la tensión y la relajación de la dominante y la tónica, la disonancia y la resolución ${ }^{9}$. Igualmente, estos compositores empezaron a cuestionar la validez del tema y desarrollo ${ }^{10}$ e iniciaron una exploración de nuevas formas de enfocar las composiciones. Surge así la tendencia a suprimir el objetivo de las progresiones armónicas, que habían proveído continuidad y organización formal por más de dos siglos. Podemos claramente identificar, no obstante, los primeros pasos firmes hacia esta serie de cambios importantes en la obra de Arnold Schoenberg, quien en 1907, como nos recuerdan Palisca y Grout, renunció “[...] al sistema de relaciones mayor-menor alrededor de un solo centro tonal que llamábamos tonalidad" (Palisca y Grout, 1996, p. 693).

Sobre Schoenberg, Palisca y Grout continúan:

Para 1923, después de seis años durante los cuales no publicó música, Schoenberg había formulado un método de composición con doce notas que se relacionan entre si. Los puntos esenciales de la teoría de esta técnica de

9 La armonía clásica contempla el uso de un centro tonal alrededor del cual, con el uso de sonidos consonantes y disonantes, se podían generar estados de tensión y relajación. El clave bien temperado de Johan Sebastian Bach (1685-1750) estableció dichas relaciones armónicas y permitió la consolidación del temperamento, a partir del cual se hace una organización de los sonidos musicales, separándolos en distancias tonales iguales (semitonos). El sistema temperado fue la base para el diseño de instrumentos temperados en el Barroco y el inicio del periodo Clásico, instrumentos que podían ejecutar piezas musicales en cualquier tonalidad sin necesidad de ser sometidos a ajustes de afinación u organización tonal no temperada, como ocurría en el Barroco temprano. El sistema temperado se convirtió en la herramienta armónica fundamental de los compositores clásicos y fue adoptado por la música popular, que llega hasta nuestros días. El sistema temperado consta de doce sonidos separados por la misma distancia tonal, contenidos en el espacio de una octava, en la que cada sonido puede ser tratado de manera enarmónica $\{$ Do-Do\#(Reb)-Re-Re\#(Mib)-Mi-Fa-Fa\#(Solb)-SolSol\#(Lab)-La-La\#(Sib)-Si\}.

10 A lo largo del período comprendido entre el Barroco tardío y el Romanticismo del siglo xix, las formas musicales se caracterizan por el desarrollo de un tema o motivo musical, una frase melódica o armónica que, a manera de repetición, sufría variaciones armónicas, melódicas o tímbricas, así como por la generación de nuevas frases, respuestas, que lo desarrollan. Por ejemplo, La quinta sinfonía de Beethoven se basa en el desarrollo de un motivo melódico compuesto por una nota (Sol) repetida en tres corcheas y otra nota (Mi) en una blanca (ta-ta-ta-tán). 
doce notas (dodecafónica) pueden ser resumidos como: la base de cada composición es una fila o serie compuesta por las doce notas o clases de tono de la octava, dispuestas en el orden que el compositor elige. Las notas de la serie deben ser usadas todas sucesivamente (como melodía) y simultáneamente (como armonía o contrapunto) sobre cualquier octava y con cualquier ritmo deseado. La fila debe ser usada no solamente en su forma original o principal, sino también en forma interválica invertida, en orden retrógrado (en reversa) o retrógrado invertido y en transposición de cualquiera de las cuatro formas. El compositor utiliza los doce tonos de la serie antes de continuar utilizando la serie en cualquiera de sus formas nuevamente. (Palisca y Grout, 1996, p. 693).

El método de Schoenberg, a la luz de las normas clásicas de composición y de su uso tradicional en la música, puede llegar a ser visto como desordenado. Sin embargo, su uso genera de cierta forma una serie de causa-efecto en la que los doce sonidos, junto con las variables de uso, componen una lista de elementos similar a una base de datos a partir de la cual el compositor construirá su obra. Como podemos notar, el método de Schoenberg ejecuta una operación similar a la construcción de una narración de la database, tal como lo pone en evidencia Manovich (2001) al inicio del milenio.

\section{Nuevas formas de notación y la indeterminación}

Así como Schoenberg agrupó los elementos de composición, las normas clásicas de composición también lo hacían, pero alrededor de la tonalidad. El método y la teoría desarrollada por Schoenberg abrieron la puerta para que la definición de música les permitiera a los compositores explorar nuevos campos sonoros alrededor del timbre, los recursos electrónicos y las nuevas tecnologías desarrolladas en la guerra. La exploración tímbrica tuvo lugar junto a la experimentación tonal y se comenzaron a introducir en la música un gran número de sonidos no acostumbrados, como los tone clusters $^{11}$ de piano, la técnica de flutter-tongue ${ }^{12}$ y glissandos armónicos $^{13}$ en

\footnotetext{
11 Se refiere musicalmente a la ejecución de varios grupos de teclas del piano al mismo tiempo. 12 Generar tremolos al hacer vibrar la lengua al mismo tiempo que se sopla para hacer sonar un instrumento de viento de manera tal que el sonido sea similar al de la pronunciación de una erre ("r").

13 Efecto sonoro que consiste en ejecutar todos los sonidos posibles en un instrumento desde una nota aguda a una nota grave.
} 
los instrumentos de viento y el col lengo ${ }^{14}$ en las cuerdas. Estos y muchos más recursos debían tener una correspondencia escrita en la partitura, razón por la cual la notación comenzó a cambiar. La notación musical encuentra en la música concreta (musique concrète), desarrollada durante la década de 1950 en Francia, su mayor necesidad de transformación. En efecto, la música concreta transformaba sonidos de la naturaleza a través de procesos mecánicos o electrónicos. Estos se ensamblaban posteriormente y se registraban en soporte magnético. Sonidos que no encuentran correspondencia en el "alfabeto" musical tradicional empezaron a poblar la gama del acto de creación musical. El solo hecho de entender la música como la organización de sonidos y silencios le permitió a algunos compositores prescindir del uso de instrumentos tradicionales, instrumentos que, recordemos, habían influido, si no exclusivamente dado forma, a la notación tradicional. La consecuencia natural de la introducción de estos instrumentos fue el desarrollo de nuevos modos de escritura, el desarrollo de una nueva notación.

Metástasis (1955), del compositor griego Iannis Xenakis (1922-2001), es la primera obra que acude a este recurso. En su partitura se puede apreciar que, en lugar de usar un pentagrama tradicional y hacer uso de las figuras tradicionales (negras, blancas, corcheas, etcétera), Xenakis diseña un diagrama gráfico en el que realiza un trazo de naturaleza geométrica: líneas rectas que insinúan diferentes alturas son subseguidas por diferentes elementos gráficos que, a manera de notación neumática, le proponen a los ejecutantes un incremento o decremento progresivo de la frecuencia, que debía iniciar desde la más baja posible hasta la más alta posible y viceversa. Para Xenakis la música ya no está contenida en un conjunto de solo doce notas dentro de una octava, sino también en cada una de las frecuencias que hay entre cada altura tradicional ${ }^{15}$.

Tanto el dodecafonismo de Schoenberg como la armonía clásica hacen uso de tan solo doce frecuencias, mientras que Xenakis, en Metástasis, propone el uso de todas las frecuencias posibles contenidas en

\footnotetext{
14 Tocar con la parte de madera del arco.

15 Quisiera aclarar al lector que las notas musicales o alturas corresponden a una frecuencia de vibración. Por ejemplo, la nota La de la tercera octava en un piano corresponde a una vibración de 440 ciclos por segundo (Hertz), su octava - La en la cuarta octava del piano-corresponde al doble, $880 \mathrm{~Hz}$. El sistema temperado separa las notas contenidas en una octava de manera logarítmica, es decir, la distancia en frecuencia entre cada semitono se incrementa en una proporción de 1/3 a medida que se acerca a la octava. Las octavas musicales no son iguales; por ejemplo, la frecuencia de la nota $D_{0}$ es 261,6 Hz, así que su octava está a 261,6 Hz de distancia, mientras que, como se vio en el caso de $L a$, la distancia con respecto a su octava es de $440 \mathrm{~Hz}$. La relación tonal entre cada octava también es logarítmica.
} 
una octava o más. Es decir, Xenakis habilita el uso de todos los sonidos posibles en un instrumento.

En la primera mitad de la década del cincuenta, Earle Brown (1926-2002) llevó la indeterminación propuesta por Xenakis más allá. En December 1952 (1952) y 4 Systems (1954), la partitura corresponde solamente al dibujo de líneas horizontales de diferente longitud y grosor, ubicadas en diferentes partes de la hoja. Para 4 Systems, los gráficos están acompañados de la siguiente instrucción: "Puede ser ejecutado en cualquier secuencia, incluso desde cualquier lado, a cualquier tempo [...] el grosor puede indicar dinámicas y clusters"16. Para December 1952, Brown anota:

Para uno o más instrumentos y/o medios de producción de sonido ... tener elementos existentes en el espacio ... espacio como una infinitud de direcciones desde una infinitud de espacios en el espacio ... para trabajar (composicionalmente y en interpretación) hacía la derecha, izquierda, atrás, adelante, arriba, abajo y todos los puntos en medio ... la partitura [el ser] una imagen de este espacio en un instante, el cual debe ser siempre considerado como irreal o transitorio ... un intérprete debe colocar todo esto en movimiento (tiempo), es decir, darse cuenta de que está en movimiento y entrar ahí ... o sentarse y dejarlo mover o moverse a través a todas las velocidades ${ }^{17}$.

En un principio, el manuscrito de la partitura de December 1952 determina una posición inicial por la ubicación de la firma del compositor que se encuentra a un borde de la hoja. Sin embargo, la partitura puede ser ubicada de cualquier manera sobre el atril. De hecho, con base en las anotaciones del compositor, muchos intérpretes han concebido la partitura como la representación tridimensional del espacio, otorgándole a las líneas más delgadas una ubicación más profunda (alejada del lector), mientras que las líneas más gruesas han sido interpretadas como las más cercanas. Es interesante notar que, en el mismo año, John Cage (1912-1992) compuso 4'33" (4 minutos 33 segundos, 1952), una pieza en la que el intérprete, que puede ser más que uno, se sienta en silencio, dándole paso a los ruidos del publico, de la sala, o los ruidos del exterior. Son estos ruidos, o silencios,

16 Brown, 4 Systems (1954), traducción propia.

17 La nota aparece en la página de un cuaderno fechado entre octubre y noviembre de 1952. Esta nota es la base de la composición December 1952, pero también es particularmente relevante para 4 Systems. 
los verdaderos compositores de la pieza. Como afirmarían Palisca y Grout (1996, p. 795), “[...] la naturaleza es el gran creador de la música”. Podríamos considerar 4'33' como una pieza que deja su material sonoro al azar, a la irregularidad de la naturaleza.

Las anteriores obras, al prescindir del uso de caligrafía musical tradicional, y al permitir una interpretación ajena al prejuicio de los grafismos, omiten tanto al instrumento musical como a su ejecutante. Esta característica, que sin lugar a dudas representa un cambio radical en la expresión musical, podría entenderse como una crítica clara al contexto tecnológico en el que aquellas expresiones musicales se manifestaban, es decir, una crítica al período de paso de la Segunda Revolución Industrial a la Tercera Revolución Industrial. Un contexto tecnológico que vio, en el campo de la música, tal como fue expuesto, el uso de toda la gama sonora propuesta por Xenakis, de los medios sonoros para producir el sonido, y su comportamiento en el espacio-tiempo postulados por Brown, y el manejo del silencio como un elemento sonoro propuesto por Cage. El ingreso de estos tres factores al proceso de creación musical hizo partícipes de la construcción del fenómeno perceptivo tanto al compositor como al intérprete, e incluso significó la inclusión plena del espectador en el proceso de creación.

\section{Espacios fractales: el minimalismo}

En 1965, Richard Wollheim emplea el término Minimal Art en un ensayo en el que expone una tesis sobre cómo el arte se había minimizado en la primera mitad del siglo xx, analizando a los neo-dadaístas, con Reindhardt a la cabeza, y los ready-made de Duchamp (Marzona, 2004). El término de Wollheim, sin embargo, se empleó después para denotar una corriente artística que surgió poco después. De forma paralela, en el campo de la pintura vanguardista en Estados Unidos, se dio una clara tendencia hacia la abstracción, que liberó definitivamente a la representación pictórica de la tradición europea (Marzona, 2004, p. 6). El título de las obras, el uso de técnicas vanguardistas, e incluso su contenido, comenzaron a ser parte de una lista de elementos comunes a los que muchos artistas recurrirían. Una primera muestra de la utilización de estos elementos se puede apreciar en Flag (1954-1955) de Jasper Johns. Flag es una pintura encáustica ${ }^{18}$ al óleo

18 Técnica pictórica en la que la cera es utilizada como aglutinante de los pigmentos, su nombre proviene del griego enkaustikos. 
sobre tela extendida sobre madera que representa la bandera de Estados Unidos con cuarenta y ocho estrellas. Más allá de ser entendida como una representación de la bandera, Johns quería que la obra fuera interpretada como la bandera en sí: como lo que es. Sin embargo, fueron los trabajos de Robert Morris (1931), Sol Lewitt (1928-2007), Donald Judd (1928-1994), Dan Flavin (1933-1996) y Carl Andre (1935) los que terminaron por llevar más allá la reflexión sobre el objeto y su representación. Fueron estos artistas quienes conformaron el cuerpo de artistas minimalistas.

En Element Series y Equivalent Series ${ }^{19}$, Carl Andre toma objetos prefabricados de formas básicas y simples y los dispone de forma repetida. La recursividad también aparece en la obra de Flavin, An Artificial Barrier of Blue, Red and Blue Fluorescent Light ${ }^{20}$. En esta obra, Flavin crea nuevos objetos a través de la disposición recursiva de tubos de neón. La recursión se materializa en la obra de Judd a través de la serialización de la producción. En el caso de Lewitt, especialmente en Serial Project No. 1 ( $A B C D)$, la reiteración de las formas cúbicas sirve de módulo base para que sean combinadas en diversos órdenes y disposiciones, generando así nuevas dimensiones del mismo objeto a partir de su repetición. Por el contrario, Morris recurre a la no repetición de formas básicas, pero las usa en grandes dimensiones ${ }^{21}$ para organizar el espacio expositivo y sumergir al espectador en él.

A partir de estas apreciaciones podríamos afirmar que el arte minimalista reúne tres elementos fundamentales:

- El uso de formas o elementos poco complejos.

- La forma reiterativa de estos elementos.

- La utilización de estos en amplios espacios o períodos de tiempo.

\section{La música minimalista}

The Tortoise (1964), de La Monte Young, es una pieza musical que reúne los tres elementos del minimalismo. A partir de la ejecución en

19 Ambas obras consistían en la disposición en el suelo de una cantidad indeterminada de baldosas o ladrillos de igual forma y dimensiones, que juntas configuraban un nuevo objeto.

20 La obra consta de una serie de bastidores. En cada uno de sus bordes se instala un tubo de neón, azul en los bordes transversales y rojo en los perpendiculares. Los bastidores, ahora cuadros de neón, se extienden a lo largo de la sala formando una barrera.

21 En Plywood Show, Morris toma grandes piezas construidas en madera contrachapada y las organiza en el espacio colgándolas, apoyándolas en las paredes o simplemente ubicándolas sobre el piso. Los objetos, a pesar de sus dimensiones y organización, permitían al observador recorrer la instalación. 
forma de pedal de una sola nota utilizando un sintetizador (un dispositivo de producción sonora cualquiera), indica a otros músicos que toquen sus instrumentos en una nota aleatoria sin ninguna referencia tonal (indeterminada) durante una larga duración de tiempo ${ }^{22}$. El resultado sonoro consiste en la combinación de timbres que conforma uno nuevo y que enmascara a los elementos básicos —iniciadores- Young no realiza giros melódicos o progresiones armónicas, las notas se mantienen por el tiempo de ejecución, repitiéndose por la naturaleza de ejecución de los instrumentos. Junto a Young, Terry Riley, Philip Glass y Steve Reich conforman la escuela minimalista de Nueva York. Acerca de los elementos que los cuatro utilizan como base para el desarrollo de sus composiciones, Wim Mertens (1983, pp. 88-89) sostiene:

Las diferencias que uno puede encontrar en las técnicas de composición que utilizan Young, Riley, Glass y Reich, de ninguna manera oscurecen las grandes similitudes en la mecánica básica de su música y sus connotaciones idelógicas. Estas se delinean más fácilmente al compararlas con el modelo musical tradicional dialéctico-romántico [...] Otra posible línea de investigación habría sido dibujar la atención sobre la influencia abierta de la música no europea, también llamada primitiva $^{23}$.

Ya que el conjunto de elementos musicales clásicos existentes antes de la guerra conforma el modelo dialéctico-romántico para Mertens, continúa afirmando:

Young y Riley son ambos discípulos del maestro de Raga India, Pandit Pran Nath. Philip Glass basó sus sistemas rítmicos en las estructuras de tiempo aditivas de las tablas de la música clásica indostaní y Steve Reich adoptó ciertos principios rítmicos de la música de Ghana y Costa de Marfil, y también del gamelan balinese. (Mertens, 1983, p. 89).

Si bien este uso de elementos musicales no puede ser tomado como el fundamento de su trabajo, hay que considerar el hecho de que los cuatro minimalistas hayan explorado expresiones culturales no Occidentales con el fin de encontrar nuevos elementos que les permitieran diferenciar

22 La versión de estreno de The Tortoise tuvo una duración de 18 minutos.

23 Las traducciones del libro de Mertens (1983) son propias. 
sus procesos en el uso del atonalismo y dodecafonismo, e incluso del indeterminismo. Esta exploración se encaminaba a la reducción de elementos musicales, reducción que apuntaba al uso de un solo elemento básico.

A continuación, se verán con detenimiento las bases de desarrollo de los primeros trabajos de estos compositores, quienes a través del uso de los tres elementos del minimalismo expuestos compusieron piezas que manifiestan ciertas características fractales.

\section{Terry Riley: In $C$ (1968).}

In $C$ se basa en la idea de reproducir bucles de cinta magnetofónica de diferentes longitudes en diferentes grabadores y de manera simultánea, generando así una yuxtaposición entre cada grabación. La partitura de In $C$ corresponde a la escritura del contenido musical que estaría grabado en cada uno de estos fragmentos de cinta. A pesar de estar organizados sobre el papel de forma secuencial y ordenada, la pieza crea su propio orden durante su ejecución, ya que cada módulo debe ser repetido por cada intérprete cuantas veces este lo desee, y cada intérprete debe seguir ordenadamente de la misma manera cada módulo. El primer módulo corresponde al pulso $^{24}$ de la pieza y debe ser ejecutado, según instrucciones de Riley, en la última octava del piano, la octava de Do. Esto lleva a Riley a construir el material melódico de cada uno de los módulos sobre esa tonalidad - Do mayor ${ }^{25}$ — , que es la tonalidad más básica del sistema, y de ahí el nombre de la obra — por la denominación de la tonalidad en inglés, " $C$ "-

La no determinación del número de intérpretes, así como la libertad para que ellos mismos sean quienes tomen la decisión de hacer el cambio de módulo durante la interpretación, tomar una pausa y generar nuevas relaciones a partir de las repeticiones, da como resultado que In $C$ no suene nunca igual. Todas las versiones de $\operatorname{In} C$ que se hayan podido tocar pertenecen a ese pequeño puñado de infinitas posibilidades sonoras.

La causa de esto son las relaciones: si bien el contorno sonoro puede ser percibido como el mismo, sus relaciones internas varían, al igual que sus puntos de referencia.

24 Quien inicie la ejecución de la pieza determinará el tempo de la misma.

25 En la música existen los modos tonales. Cada uno se construye a partir de cada una de las notas musicales y se configura con una estructura tonal determinada. Do mayor es el modo tonal más básico y simple, corresponde a tocar todas las teclas blancas contenidas en una octava de Do. No representa mayor dificultad para el intérprete, pues no posee alteraciones (bemoles o sostenidos). 


\section{Philip Glass: Music in Fifths (1969).}

Inicialmente concebida para ser ejecutada en un instrumento de teclado, la pieza se basa en el movimiento melódico armónico paralelo ${ }^{26}$ de quintas justas $^{27}$. Transgrediendo el uso del intervalo dentro de la armonía clásica ${ }^{28}$, el resultado sonoro de Music in Fifths es similar a la ejecución repetitiva de varios fragmentos de melodía que incrementan su longitud a lo largo del tiempo. La sonoridad de la quinta es simple, hueca por dentro, y en extensos periodos de tiempo se hace inaudible.

La partitura de Music in Fifths está organizada de manera lineal. De nuevo, hay un orden determinado para la ejecución de los conjuntos de sonidos. En la partitura, Glass utiliza la línea del pentagrama solamente para darle al intérprete el orden en el que se deben hacer las repeticiones. Sin embargo, el intérprete es libre de decidir el número de veces que repite cada módulo. Estos módulos se diferencian porque cada uno tiene una o varias notas adicionales con respecto al anterior, lo cual incrementa progresivamente su longitud. Además, Glass suma la adición y la sustracción a la repetición. En sus palabras, su propósito es "crear nuevas estructuras con la asistencia de sonidos" (Mertens, 1983, p. 71). Al respecto de la adición y sustracción dice:

Que el oyente en este asunto está un paso más allá de mí. Mientras que a mí, como compositor, me preocupa la estructura y la unidad formal, la atención de mi audiencia está principalmente concentrada en el sonido como tal. Debería concentrarme más en el resultado sonoro que en los aspectos formales. (Mertens, 1983, p. 71).

26 Armónicamente en música, existen tres tipos de movimientos. Estos movimientos solo se dan en una relación de dos líneas melódicas ejecutadas simultáneamente. El movimiento paralelo corresponde a un movimiento en la misma dirección manteniendo el mismo intervalo. El movimiento contrario corresponde a un movimiento en diferente dirección generando nuevos intervalos en el tiempo. Finalmente, el movimiento directo o similar se da hacia la misma dirección pero cambiando el intervalo. En el caso de Glass, las dos melodías (voces), ejecutadas una por la mano derecha y otra por la mano izquierda en el piano, se mueven de forma paralela en un intervalo de quinta justa.

27 Intervalo armónico que corresponde al número de alturas que separan un tono de otro. Por ejemplo, las notas Do-Sol configuran un intervalo de quinta, pues entre ellas hay una separación de cinco notas, incluyéndolas. Do (Re-Mi-Fa) Sol.

28 En la armonía de la música sacra en el Barroco, especialmente la música coral, se restringía el movimiento paralelo de intervalos perfectos (cuarta, quinta y octava). La realización de este tipo de movimientos, por tener una sonoridad distintiva, consonante, frente a la carga disonante que otros intervalos pueden tener, generaba la sensación de desaparición de una de las voces, lo que llevó a que su uso estuviera restringido en los estudios de la armonía clásica entre el Barroco y el Romanticismo. 
Glass muestra en sus propias palabras el valor del contorno, sin desconocer el poder que cada elemento que lo conforma —en este caso el módulo sonoro- tiene sobre dicho contorno envolvente. El control que el intérprete tiene sobre este pequeño elemento es vital para el funcionamiento de la obra. Podríamos decir que Glass hace una fractalización de la pieza, ya que en ella se resaltan los factores heterogéneos de las partes que la componen y, sin embargo, en su forma heterogénea se crea una armonía (Duarte, 2014, pp. 252-254).

En una obra sucesiva que incluye Music in Contrary Motion (1969) y Music in Similar Motion (1973), Glass usa de manera más clara la repetición, adición y sustracción, pero esta vez con módulos compuestos por los respectivos movimientos armónicos. En la película Tango (1983) de Zbigniew Rybszcynski, se encuentra el mismo proceso realizado por Glass. Ambas obras adicionan progresivamente, sobre el mismo plano, elementos que se van a repetir. En ambas estructuras el patrón corresponde a la aparición de un nuevo elemento inmediatamente después de la repetición del anterior, lo cual genera relaciones espaciales que configuran una estructura narrativa. Sobre este fenómeno narrativo, Duarte afirma, analizando Tango, que:

Por un lado, el espectador de esta película no percibe la sucesión debido a la yuxtaposición de diferentes espacios, espacios que presentan su propio tiempo. Por otro lado, la coexistencia de diferentes tiempos en el espacio, y su recursividad, elimina por completo la posibilidad de ubicar al complexus $^{29}$ en una coordenada especifica de tiempo para así poder proyectar el conjunto. (Duarte, 2014, p. 253).

\section{Steve Reich: It's Gonna Rain (1965), Come Out (1966).}

Quisiera iniciar el análisis de la siguiente obra citando las palabras con las que Reich describe el evento que generó la pieza:

Para finales de 1964, grabé una cinta en Union Square, en San Francisco, de un predicador de color, el hermano Walter, predicando acerca del Gran Diluvio. Estaba extremadamente impresionado con la calidad melódica de su discurso, el cual parecía estar al borde del canto.

29 El complexus define un grupo compuesto por factores heterogéneos que forman un sistema complejo. Cada factor, sin perder su heterogeneidad, hace parte de un complejo sistema armónico y, en consecuencia, aparentemente homogéneo. 
En 1965, comencé a hacer bucles de cinta de su voz grabada, lo que hizo que la calidad musical de su discurso emergiera aún con más fuerza. [...] Mi interés original en la música electrónica era la posibilidad de trabajar con discurso grabado. [...] Recuerdo decepcionarme cuando la música para cinta, o música concreta como se llama, normalmente presentaba sonidos que no se podían reconocer, cuando lo que parecía interesante para mí era que una grabadora de cinta grababa sonidos reales como los de un discurso, tal y como una cámara cinematográfica graba imágenes reales. Si uno puede presentar ese discurso sin alterar su altura o su timbre, uno mantendría el poder emocional que el discurso tiene mientras intensifica su melodía y significado a través de la repetición y el ritmo. [...] La idea de usar la repetición constante creció parcialmente trabajando con bucles de cinta en 1963, pero principalmente ayudando a Terry Riley a preparar la primera presentación, en 1964, de su obra In $C$, en la que diferentes patrones repetitivos se combinaban simultáneamente. (Reich, 2002, p.19 ${ }^{[30]}$.

Steve Reich encontró en sus ejercicios de grabación algunos medios de producción sonora alternativos a los que Riley y Glass venían utilizando. $\mathrm{Su}$ principal problema se centraba en poder hacer uso de la repetición constante como técnica musical y la forma en que él mismo pudiera usar esa forma con el material que poseía.

"Mi primer pensamiento fue reproducir el bucle de cinta junto a una copia del mismo en alguna relación particular" (Reich, 2002, p. 20), lo que implica lógicamente dos reproductores. Reich continúa describiendo el proceso con estas palabras: "Descubrí que lo más interesante de todo, musicalmente, fue simplemente alinear los bucles al unísono dejándolos cambiar lentamente de fase ${ }^{31}$ entre sí” (Reich, 2002, p. 20).

Estos progresivos cambios de fase se perciben al inicio como un barrido de frecuencia que poco a poco se empieza a convertir en una corta reverberación para llegar a ser un eco perceptible en el que, en el caso de It's Gonna Rain (1965), se conforma un contrapunto silábico dentro de una misma frase: "It’s gonna/It’s gonna rain/rain".

\footnotetext{
30 Las traducciones del libro de Reich (2002) son propias.

31 Las máquinas reproductoras de sonido con cinta magnetofónica funcionan con un motor que permite que la cinta recorra entre 15 y 30 pulgadas por segundo. A medida que el material magnetofónico pasa de un carrete a otro, por el peso, estas velocidades se ven alteradas, modificando la precisión en la velocidad del reproductor. En el caso de Reich, esto genera una entropía en la sincronización de los dos reproductores que produce los cambios de fase.
} 
Cuando escuché eso me di cuenta de que era mucho más interesante que cualquier otra relación en particular, porque era el proceso de pasar gradualmente por todas las relaciones canónicas haciendo una pieza entera y no solo un momento en el tiempo (Reich, 2002, p. 21).

Reich aplica la misma técnica en Come Out ${ }^{32}$ (1966), la última pieza basada en grabaciones hechas por él antes de empezar a escribir música instrumental. Cabe recordar que es en el campo de la música instrumental en donde Reich utiliza como técnica musical el cambio de fase. En Piano Phase, por ejemplo, dos pianistas inician una ejecución al unísono del mismo patrón melódico, compuesto por notas escritas en semicorcheas. De acuerdo con la instrucción de Reich, uno de los dos pianistas acelera el tempo hasta que la relación rítmica se haya desplazado el valor de una semicorchea. Este desplazamiento genera un patrón nuevo, de la misma forma que ocurría con las sílabas en el trabajo con cinta. En Piano Phase, el pianista que tiene el papel de generar el desfase del patrón debe continuar haciéndolo hasta haber recorrido todas las relaciones posibles, momento en el cual se puede finalizar la ejecución (Reich, 2002, p. 22). Las relaciones, o estructuras canónicas, como las llama Reich, se establecen a partir de los conceptos teóricos tradicionales del dialécticoromántico y constituyen el elemento básico del material musical de Reich.

\section{Conclusión}

En el volumen sobre la narración fractal, Duarte analiza el fenómeno de retroalimentación del video ${ }^{33}$ y, a través de este, explora la fractalización de un espacio narrativo en el que el iniciador y generador se repiten infinitamente (Duarte, 2014, p. 87). Como se vio, la música minimalista usa también patrones recursivos que generan, de cierta forma, una relación similar entre iniciador y generador. En el caso de Reich, el cambio de fase entre un patrón melódico y su duplicado en el bucle de cinta genera un efecto sonoro similar a la video retroalimentación ${ }^{34}$. De igual forma

32 "I had to, like, open the bruise up and let some of the bruise blood come out to show them". Esta frase se repite tres veces y es sometida a fragmentación a través del cambio de fase. En ese sentido, la pieza se desarrolla desde el unísono hacia una incomprensible superposición de fragmentos fonéticos de un texto (Mertens, 1983).

33 Es el fenómeno que resulta cuando una cámara de video encuadra su propia información visual en el monitor. En otras palabras, la cámara ve su propia imagen encuadrada (Duarte, 2014).

34 Es el fenómeno que resulta cuando un micrófono captura su propia información sonora desde un altavoz. En otras palabras, el micrófono escucha su propio sonido. 
ocurre en In $C$, donde la indeterminación de las relaciones se repite en cada módulo, y estas son infinitas. Esta pieza correspondería a un sistema recursivo que permite la operación $\mathrm{X}+\mathrm{n}$ y, en consecuencia, permite la creación del infinito (Duarte, 2014, p. 87). Las analogías entre el espacio concebido por el minimalismo y el espacio fractal van aún más allá si consideramos que, tal y como sucede en la topología, como lo menciona Duarte, un objeto cambia su carácter cuando es colocado en el espacio de constante movimiento infinito, y ahí, en ese espacio creado por el objeto, cada patrón asume una función. Los fragmentos sonoros construidos tanto de manera canónica como en las piezas de Riley y Glass, o aquellas piezas producto del desfase, constituyen las dinámicas internas de cada obra y corresponden al objeto que modifica su comportamiento al ingresar en el sistema de repetición, adición o sustracción. En el minimalismo, de forma contraria a lo que sucede con las formas clásicas, a los fragmentos melódicos no se les trata como motivo para la construcción de un tema y su desarrollo, sino que se los denomina 'patrón'. El patrón es un objeto independiente, iniciador de la repetición, que durante el tiempo genera relaciones entre él mismo, creando así objetos nuevos que también se repiten y hacen parte, a su vez, de un objeto más grande: la obra. "Es una característica de la música repetitiva que no se exprese nada: se mantiene solo por sí misma” (Mertens, 1983, p. 88).

Aquel comerciante de finales del siglo xix al accionar la manivela del órgano portátil, y al escuchar una vez tras otra cada una de las repeticiones de esa melodía, no solo la deformaba, también la convertía en un patrón. El detalle de su contenido melódico dejaba de ser esencial para su funcionamiento y, una vez convertido en patrón, cada repetición, generada por los giros del cilindro codificado, adquiría una función dentro del sistema producido por las repeticiones. Este sistema, gracias a su continua generación, gracias a su devenir, manifestaba el paso del objeto al objectil (Deleuze, 1984), el cual es puesto en evidencia por Duarte con estas palabras: "Un objeto ya no es un objeto cuando se coloca en un continium" (Duarte, 2014, p. 190).

Es interesante recordar que, de cierta manera, el campo audiovisual, aunque comprendido como una sola dimensión, fisiológicamente determina algunas diferencias ontológicas. En mi parecer, el concepto de audiovisión no puede comprender la narración audiovisual como una dimensión única y homogénea. Tal como es analizada por Michel Chion, 
la percepción visual y la percepción auditiva tienen su propio ritmo promedio natural; básicamente, el oído analiza, procesa y sintetiza más rápido que el ojo. [...] El movimiento rápido, a nivel visual, no formará una figura distintiva, su trayectoria no entrará en la memoria en forma de una imagen precisa, en cambio, en la misma longitud de tiempo la trayectoria sonora tendrá éxito en delinear de forma clara y definida, individualizado, reconocible y distinguible de otros. (Chion, 1994, p. 11)

Es interesante, con respecto a esto, notar que Rybsczynski requirió solamente de ocho minutos para realizar una organización espacial compleja como la de Tango. Por el contrario, Riley necesitó cuarenta y dos minutos para realizar un espacio análogo en In C. Por su lado, Glass necesitó treinta en Music in Fifths y Reich, como fue analizado anteriormente, desarrolló las fases de Come Out en trece minutos. Piano Phase, por su parte, requiere de veinte minutos. Si bien lo importante es el contorno o la envolvente, en el campo de la música se necesita más material y un mayor tiempo para desarrollar los espacios recién estudiados. En el campo auditivo, el oído puede aislar un detalle y seguirlo de forma sostenida en el tiempo. Sin embargo, la creación de los espacios sonoros fractales requiere importantes cantidades de tiempo para poder desarrollar los detalles necesarios y recursivos.

\section{Referencias}

Chion, M. (1994). Audio-Vision Sound on Screen. Nueva York: Columbia University Press.

Deleuze, G. (1978). La imagen-tiempo. Barcelona: Paidós Ibérica.

Deleuze, G. (1984). La imagen-movimiento. Barcelona: Paidós Ibérica.

Duarte, G. A. (2014). Fractal Narrative: About the Relationship Between Geometries and Technology and Its Impact on Narrative Spaces. Bielefeld: Transcript.

Manovich, L. (2001). The Language of New Media. Massachusetts, Estados Unidos: The Mit Press.

Marzona, D. (2004). Arte minimalista. Estados Unidos: Taschen.

Mertens, W. (1983). American Minimal Music: La Monte Young, Terry Riley, Steve Reich, Philip Glass. Londres: Kahn \& Averill.

Palisca, C. y Grout, D. (1996). A History of Western Music. Nueva York: Norton.

Reich, S. (2002). Writings on Music. Nueva York: Oxford University Press. 